\title{
Constant- $q$ data representation in Neutron Compton scattering on the VESUVIO spectrometer
}

\author{
R. Senesi ${ }^{a, b, *}$, A. Pietropaolo ${ }^{a, b}$, C. Andreani ${ }^{a, b}$ \\ a Dipartimento di Fisica, Università degli Studi di Roma "Tor Vergata”, Via della Ricerca Scientifica 1, 00133 Roma, Italy \\ ' Centro NAST, Nanoscienze \& Nanotecnologie \& Strumentazione, Università degli Studi di Roma "Tor Vergata", Via della Ricerca Scientifica 1, 00133 Roma, Italy
}

\section{A R T I C L E I N F O}

\section{Article history:}

Received 2 March 2008

Received in revised form

24 June 2008

Accepted 26 June 2008

Available online 3 July 2008

Keywords:

Deep inelastic neutron scattering

Inverse-geometry neutron spectrometers

\begin{abstract}
A B S T R A C T
Standard data analysis on the VESUVIO spectrometer at ISIS is carried out within the Impulse Approximation framework, making use of the West scaling variable $y$. The experiments are performed using the time-of-flight technique with the detectors positioned at constant scattering angles. Line shape analysis is routinely performed in the $y$-scaling framework, using two different (and equivalent) approaches: (1) fitting the parameters of the recoil peaks directly to fixed-angle time-of-flight spectra; (2) transforming the time-of-flight spectra into fixed-angle $y$ spectra, referred to as the Neutron Compton Profiles, and then fitting the line shape parameters. The present work shows that scattering signals from different fixed-angle detectors can be collected and rebinned to obtain Neutron Compton Profiles at constant wave vector transfer, $q$, allowing for a suitable interpretation of data in terms of the dynamical structure factor, $S(q, \omega)$. The current limits of applicability of such a procedure are discussed in terms of the available $q$-range and relative uncertainties for the VESUVIO experimental set up and of the main approximations involved.
\end{abstract}

(c) 2008 Elsevier B.V. All rights reserved.

\section{Introduction}

Studies of momentum distribution in condensed matter systems provide an insight into single-particle dynamical properties, probing single-particle potentials and wave functions, allowing in several cases a determination of the Born-Oppenhimer potential [1]. Deep Inelastic Neutron Scattering (DINS) is a unique technique to probe the short-scale (time and distance) dynamics, measuring the single-particle momentum distribution $n(p)$ and mean kinetic energy, $\left\langle E_{\mathrm{K}}\right\rangle[1,2]$. The VESUVIO spectrometer, at the ISIS spallation neutron source, has been specifically designed for DINS measurements in a wide kinematical space: $1 \mathrm{eV}<\hbar \omega<$ $30 \mathrm{eV}$ and $20 \AA^{-1}<q<600 \AA^{-1}[3,4]$. Measurements of momentum distribution and mean kinetic energy have been carried out on a variety of systems ranging from ${ }^{4} \mathrm{He}$ in bulk and confined geometries [5,6], liquid ${ }^{3} \mathrm{He}-{ }^{4} \mathrm{He}$ mixtures [7], water in confined geometries [8,9], protein hydration shells [10], proton conducting systems [11], amorphous systems and strong H-bonds [12,13]. At the same time, a strong research and development activity is carried out for eV energy analysis [14], detection systems [15] and for the accurate determination of the spectrometer's resolution [16].

\footnotetext{
* Corresponding author at: Dipartimento di Fisica, Università degli Studi di Roma “Tor Vergata", Via della Ricerca Scientifica 1, 00133 Roma, Italy.

Tel.: +3906 72594549; fax: +39062023507.

E-mail address: roberto.senesi@roma2.infn.it (R. Senesi).
}

In the present work, it is shown that signals from several fixedangle detectors can be collected and rebinned to obtain Neutron Compton Profiles at constant wave vector transfer, $q$. An application to the case of proton momentum distribution measurements will be discussed in detail. This has the advantage that the experimental response function at constant $q$ depends only on the magnitude of the chosen $q$ and not on the particular $(y, q(y))$ kinematic path of the particular fixed-angle detector. Moreover, an interpretation of data in terms of the dynamical structure factor, $S(q, \omega)$, can be carried out for a suitable comparison with theoretical models.

\section{DINS fundamentals}

In the DINS regime, the scattering is well described within the framework of the Impulse Approximation (IA), which is exact in the limit of infinite momentum transfer $[1,2]$. This assumes that a single particle of the system, struck by the scattering probe, recoils freely from the collision, with inter-particle interaction in the final state being negligible (i.e., the wave function of the particle in its final state is a plane wave). Under these conditions, applying the momentum and energy conservation laws, the energy, $\hbar \omega$, and momentum, $\hbar \mathbf{q}$, transfers are related by

$$
\hbar \omega=\frac{\left(\mathbf{p}_{\mathbf{n}}-\mathbf{p}_{\mathbf{n}}^{\prime}\right)^{2}}{2 M}+\frac{\left(\mathbf{p}_{\mathbf{n}}-\mathbf{p}_{\mathbf{n}}^{\prime}\right) \cdot \mathbf{p}}{M}
$$


where $M$ and $\mathbf{p}$ are the mass and the momentum of struck particle before collision, while $\mathbf{p}_{\mathbf{n}}$ and $\mathbf{p}_{\mathbf{n}}^{\prime}$ are the momenta of the incident and scattered neutron (i.e., $\mathbf{p}_{\mathbf{n}}-\mathbf{p}_{\mathbf{n}}^{\prime}=\hbar \mathbf{q}$ ), respectively. Thus, the energy distribution of the scattered neutrons is directly related to the distribution of particle momenta parallel to the wave vector transfer. Within the framework of the IA, the dynamical structure factor is described by

$S_{\mathrm{IA}}(\mathbf{q}, \omega)=\hbar \int n(\mathbf{p}) \delta\left[\hbar \omega-\hbar \omega_{\mathrm{r}}-\frac{\mathbf{p} \cdot \hbar \mathbf{q}}{M}\right] \mathrm{d} \mathbf{p}$

where $n(\mathbf{p})$ is the atomic momentum distribution and $\hbar \omega_{\mathrm{r}}$ is the recoil energy:

$\hbar \omega_{\mathrm{r}}=\frac{\hbar^{2} q^{2}}{2 M}$.

The physical implication of Eq. (2.2) is that scattering occurs between the neutron and a single particle with conservation of kinetic energy and momentum of the particle + neutron system, as introduced in Eq. (2.1).

If the IA is valid, the two dynamic variables $\omega$ and $q$ can be explicitly coupled through the definition of the West scaling variable $y$ as [2]

$y=\frac{M}{\hbar^{2} q}\left(\hbar \omega-\hbar \omega_{\mathrm{r}}\right)$.

Eq. (2.2) can be then reduced to the form

$S_{\mathrm{IA}}(\mathbf{q}, \omega)=\frac{M}{\hbar q} J(y, \hat{q})$

where

$J(y, \hat{q})=\hbar \int n\left(\mathbf{p}^{\prime}\right) \delta\left(\hbar y-\mathbf{p}^{\prime} \cdot \hat{q}\right) \mathrm{d} \mathbf{p}^{\prime}$.

$J(y, \hat{q})$ is the Neutron Compton Profile and is formally the Radon transform of the momentum distribution. The quantity $\hat{q}$ is a unit vector, as $J(y, \hat{q})$ no longer depends on the magnitude of $\mathbf{q}$. The function $J(y, \hat{q})$ d $y$ represents the probability for an atom to have a momentum parallel to $\hat{q}$ of a magnitude between $\hbar y$ and $\hbar(y+\mathrm{d} y)$.

In an isotropic system, the direction $\hat{q}$ is immaterial and Eq. (2.6) becomes

$J(y)=2 \pi \hbar \int_{|\hbar y|}^{\infty} p n(p) \mathrm{d} p$.

The deviations from the IA which occur at finite $q$ are generally referred to as Final State Effects (FSE). FSE are due to the localization of the struck atom in its final state, by surrounding atoms. These make the momentum in the final state uncertain and since the dynamic structure factor, in Eq. (2.2), is determined by Fourier transform of the product of initial and final state wave functions, the momentum in the initial state is also uncertain. The overall effect is a broadening of the observed $J(y)$, which is similar to that introduced by an instrumental resolution effect. When FSE are present, the Neutron Compton Profile becomes $q$ dependent, i.e., $J(y)$ needs to be replaced by $J(y, q)$, so that Eq. (2.5) is replaced by

$J(y, q)=\frac{\hbar q}{M} S(q, \omega)$.

Representing the FSE as a series in powers of $1 / q$, one finds for the isotropic case according to Sears [17]:

$J(y, q)=J(y)-\frac{A_{3}}{q} \frac{\mathrm{d}^{3}}{\mathrm{~d} y^{3}} J(y)+\frac{A_{4}}{q^{2}} \frac{\mathrm{d}^{4}}{\mathrm{~d} y^{4}} J(y)+\cdots$

where the coefficients $A_{\mathrm{n}}$ are related to the interatomic potential [17]. FSE can be strongly reduced by working at high wave vector transfers, typically above $30 \AA^{-1}$. The expansion above has been proven to be equivalent to the Convolution Approach, in which a final state function is convoluted to the asymptotic $J(y)$ to obtain
$J(y, q)$, for various systems such as solid helium [18,19] and hydrogen-containing systems [12]. It has to be noted that for proton studies on VESUVIO, FSE corrections are generally found to be not observable beyond the first asymmetric $1 / q$ term.

\section{Standard data analysis on VESUVIO}

On VESUVIO, the experiments are carried out using the timeof-flight technique with the detectors positioned at constant scattering angles. Neutron energy analysis is performed via resonance absorption filters placed in the secondary flight path between sample and detectors. Therefore, the final neutron velocity and energy are related by the expression $E_{1}=m v^{2} / 2$, where $m$ is the neutron mass. The energy of the incident neutron is determined from a measurement of the neutron time-of-flight via the equation

$t=\frac{L_{0}}{v_{0}}+\frac{L_{1}}{v_{1}}$

where $t$ is the measured time-of-flight, $L_{0}$ and $L_{1}$ are the lengths of the incident and the scattered flight paths of the neutron and $v_{0}$ and $v_{1}$ are the velocities of the incident and scattered neutrons. The energy transfer is

$\hbar \omega=m \frac{\left(v_{0}^{2}-v_{1}^{2}\right)}{2}$

and the momentum transfer

$\hbar q=m\left(v_{0}^{2}+v_{1}^{2}-2 v_{0} v_{1} \cos \phi\right)^{1 / 2}$

where $\phi$ is the scattering angle. The relationship between time-offlight spectra and response function is then [20]

$$
\begin{aligned}
C(t, \phi)= & A \int_{-\infty}^{\infty} \mathrm{d} \tau \int_{0}^{\pi} \mathrm{d} \varphi \int_{0}^{\infty} \mathrm{d} L_{0} \\
& \times \int_{0}^{\infty} \mathrm{d} L_{1} P\left(\tau, \varphi, L_{0}, L_{1}\right) \int_{0}^{\infty} \mathrm{d} E_{0} \Phi\left(E_{0}\right) \\
& \times \int_{0}^{\infty} \mathrm{d} E_{1} \eta\left(E_{1}\right)\left[1-T_{\mathrm{Au}}\left(E_{1}\right)\right]\left(\frac{E_{1}}{E_{0}}\right)^{1 / 2} \frac{J(y, q)}{q} \\
& \times \delta\left[t-\tau-L_{0}\left(\frac{m}{2 E_{0}}\right)^{1 / 2}-L_{1}\left(\frac{m}{2 E_{1}}\right)^{1 / 2}\right]
\end{aligned}
$$

where $A$ is a constant depending on both the instrument and the sample, $\tau$ is the time delay due to the moderator width, $\varphi$ is the actual scattering angle (whose mean value is the nominal scattering angle of the detector considered, $\phi), P\left(\tau, \varphi, L_{0}, L_{1}\right)$ is the distribution of the four aforementioned random variables, $E_{0,1}$ are the initial (final) neutron energies, $\Phi\left(E_{0}\right)$ is the incoming neutron flux, $\eta\left(E_{1}\right)$ is the detection efficiency, $T_{\mathrm{Au}}$ is the transmission of the (gold) analyzer foil, $q$ and $y$ are also functions of the actual values of $\left(\varphi, E_{0}, E_{1}\right)$. However, experimental data are usually transformed into effective spectra at constant angle via the mean values of $\left(\tau, \varphi, L_{0}, L_{1}, E_{1}\right)$, i.e. $\left(\bar{\tau}, \phi, \bar{L}_{0}, \bar{L}_{1}, \bar{E}_{1}\right)$, known from the VESUVIO calibration [1]

$J_{\exp }^{(\phi)}(y, q(y))=\frac{B M}{E_{0} \Phi\left(E_{0}\right)} Q C(t, \phi)$

where $B$ is another constant depending on both the instrument and the sample and $M$ is the sample atomic mass. $J_{\exp }^{(\phi)}(y, q(y))$ is usually represented as $F^{(\phi)}(y, q(y))$. For fixed-angle spectra, $y$ (and then $q(y))$ is considered as function of $E_{0}$ only, $\left(\phi, \bar{E}_{1}\right)$ being fixed parameters, and through the approximate time-of-flight equation

$t \simeq \bar{\tau}+\bar{L}_{1}\left(\frac{m}{2 \bar{E}_{1}}\right)^{1 / 2}+\bar{L}_{0}\left(\frac{m}{2 E_{0}}\right)^{1 / 2}$

$y$ and $q(y)$ become function of $t$. Within the framework of the Convolution Approximation [12], the experimental response 
function is then expressed by the convolution of $F^{(\phi)}(y, q(y))$ with the fixed-angle spectrometer resolution function, $R^{(\phi)}(y, q(y))$, the latter being determined by calibration procedures and Monte Carlo neutron transport simulations [21,22]:

$F^{(\phi)}(y, q(y)) \simeq \int \mathrm{d} y^{\prime} \int \mathrm{d} q^{\prime} J\left(y^{\prime}, q^{\prime}\left(y^{\prime}\right)\right) R^{(\phi)}\left(y-y^{\prime}, q^{\prime}(y)\right) \delta\left(q(y)-q^{\prime}\right)$.

Standard data analysis procedures consist of the determination of the line shape parameters of the fixed-angle experimental response functions (3.7) and, consequently, of the momentum distribution, $n(p)$. Each response function results from the contribution of the asymptotic response, or the longitudinal momentum distribution, $J(y)$, and the $q(y)$ dependent additive corrections with values of $q(y)$ varying across the recoil peak.

Indeed, a typical fixed-angle time-of-flight spectrum is recorded in the range $50 \mu \mathrm{s} \leqslant t \leqslant 500 \mu \mathrm{s}$ with constant time bins of $0.5 \mu$ s, i.e., 900 time channels. We stress that this corresponds, for each of the 32 detector elements, to 900 different values of energy transfers, $\hbar \omega$, wave vector transfers, $q$, and longitudinal momenta, $y$. In particular, in the case of light scattering masses such as hydrogen, the range of wave vector transfers extends up to approximately $600 \AA^{-1}$, for scattering angles close to $90^{\circ}$ and short time of flights. Recent alternative data analysis approaches have been envisaged, within the context of the measurements of the so-called "intensity deficit", making use of the Waller-Froman Jacobian of the transformation of the time-of flight data from a constant scattering angle scan, into a constant momentum transfer scan [23-25]. However, the latter implies an exact energy-wave vector dispersion relation which is valid only at the center of the recoil peak; moreover, a precise relationship between the width of the experimental response function at constant angle and at the "equivalent" constant- $q$ spectra can be carried out assuming only a Gaussian momentum distribution, thus neglecting the non-Gaussian details characterizing, for example, proton momentum distribution in several hydrogencontaining systems [1].

\section{Kinematic range for proton momentum distribution experiments}

In the case of measurements of hydrogen-containing samples, a typical experimental set up consists of $32{ }^{6} \mathrm{Li}$ glass detector elements placed in the angular range $32^{\circ} \leqslant \phi \leqslant 67^{\circ}$. Gold foil analyzers are used to select the final energy, whose mean value is $\bar{E}_{1}=4908 \mathrm{meV}$. The corresponding kinematic $(q, \hbar \omega)$ range can be calculated from Eqs. (3.2) and (3.3), and is reported in Fig. 1, together with the corresponding proton recoil line. We stress that, for the whole array of fixed-angle detectors, typically $900 \times 32$ different $q$ and $900 \times 32$ different $\hbar \omega$ values are accessed. Examples of $F^{(\phi)}(y, q(y))$ for two fixed-angle detectors are reported in Figs. 2 and 3. For the whole set of detectors, the corresponding fixed-angle $q(y)$ values are shown in Fig. 4.

\section{Normalization of Neutron Compton Profiles}

In order to compare data from different detectors, a common procedure is to calibrate each fixed-angle detector with time-offlight, thus energy, dependent calibration measurements [26]. In the case of DINS measurements, once the standard data reduction has been carried out, which includes correction for multiple scattering, absorption and scattering from heavier mass such as sample container, it is possible to relate the single detector

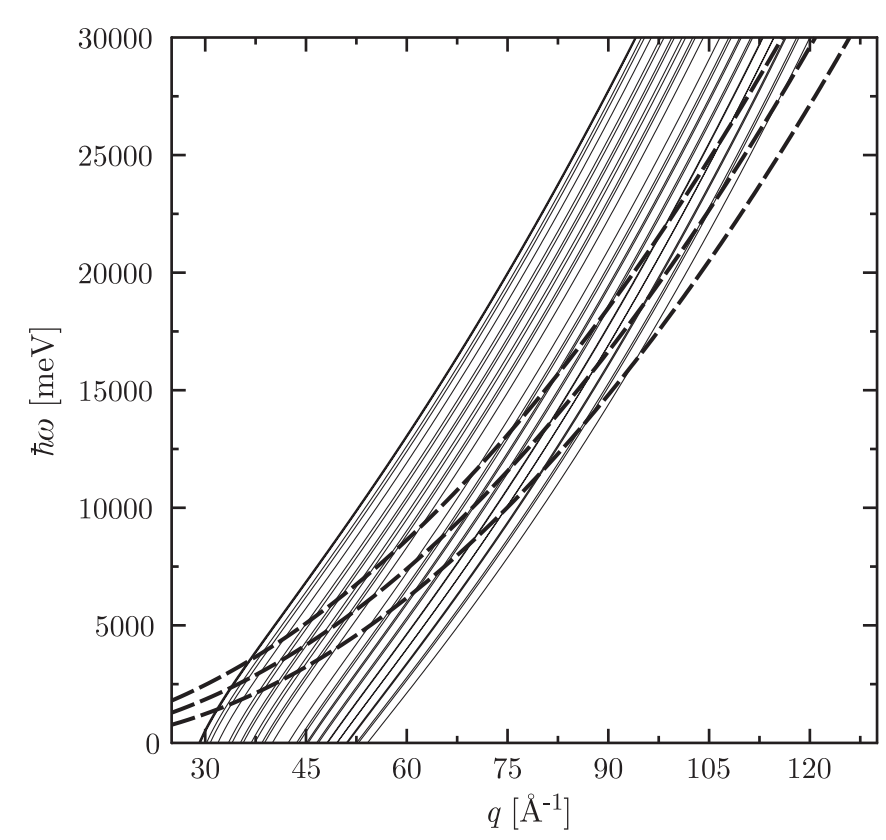

Fig. 1. Typical fixed-angle $q$, $\hbar \omega$ values for proton momentum distribution for detectors in the angular range $32^{\circ} \leqslant \phi \leqslant 67^{\circ}$ (continuous lines); proton recoil line with a line width of $5 \AA^{-1}$ is also reported (dashed lines).

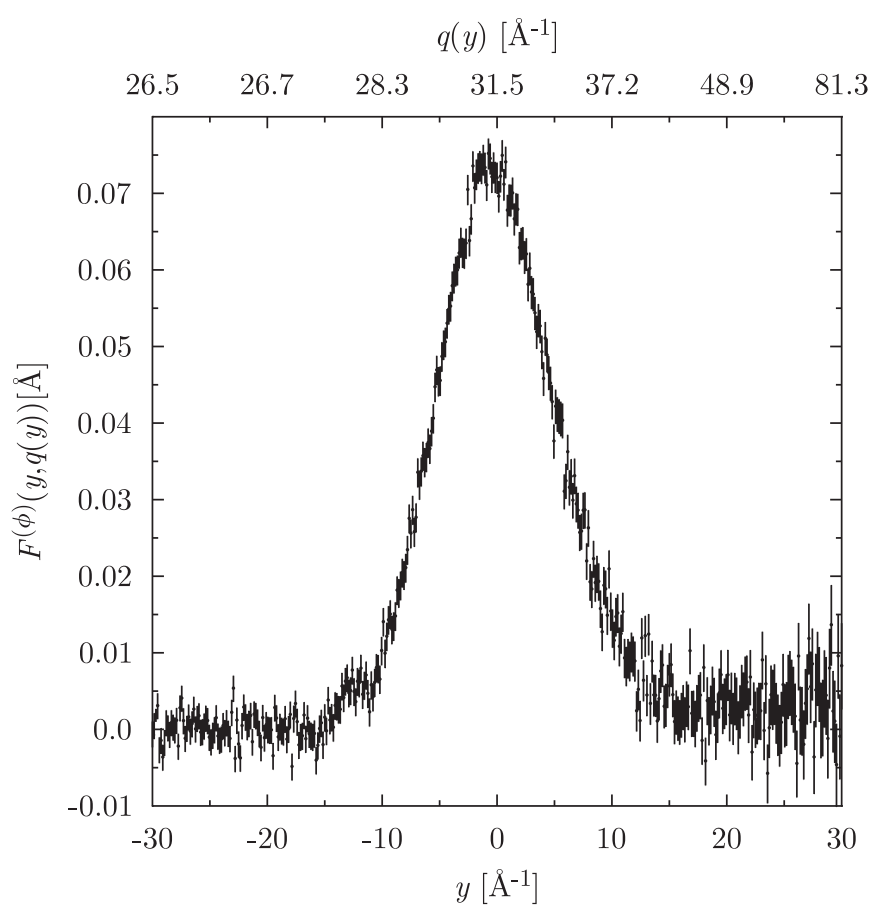

Fig. 2. Example of $F^{(\phi)}(y, q(y))$ for a fixed detector at $34.1^{\circ}$ for a sample of bulk water at $300 \mathrm{~K}$. The corresponding $q(y)$ values are on the upper $x$-scale.

intensities considering the following sum rules for $J(y)$ [17]:

$\int_{-\infty}^{\infty} \mathrm{d} y J(y)=1$
$\int_{-\infty}^{0} \mathrm{~d} y J(y)=\frac{1}{2}$.

Following Eq. (2.9) it is possible to express $J(y, q)$, at constant $q$, as the sum of the asymptotic part, $J(y)$, and the FSE term, $\Delta J(y, q)$, i.e., 


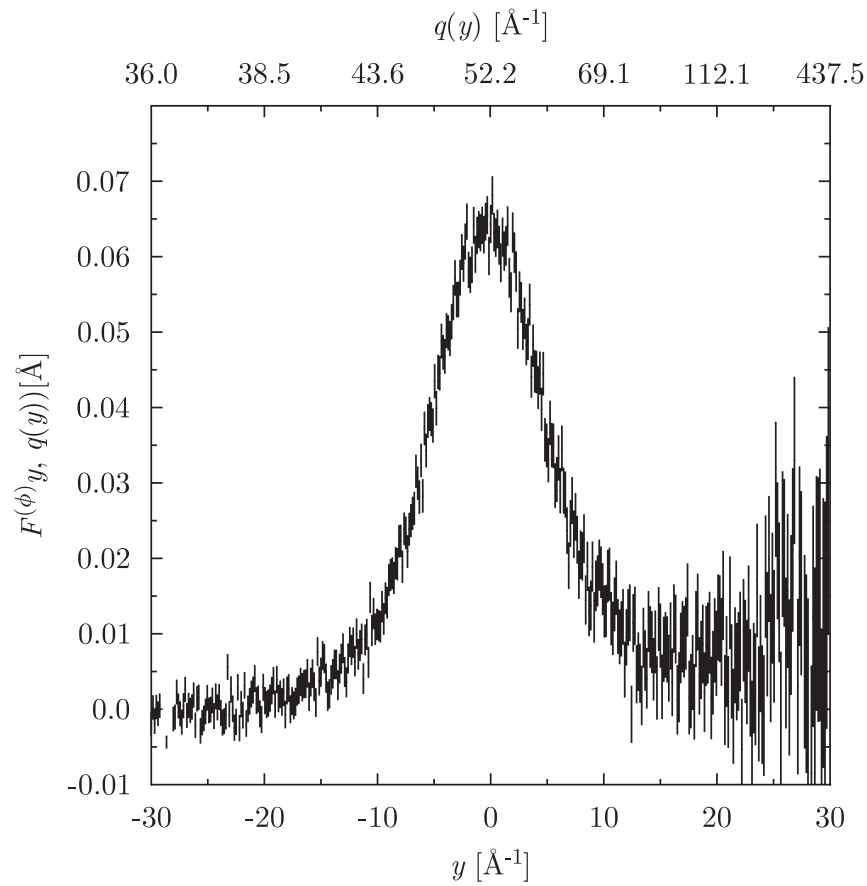

Fig. 3. Example of $F^{(\phi)}(y, q(y))$ for a fixed detector at $48.1^{\circ}$ for a sample of bulk water at $300 \mathrm{~K}$. The corresponding $q(y)$ values are on the upper $x$-scale.

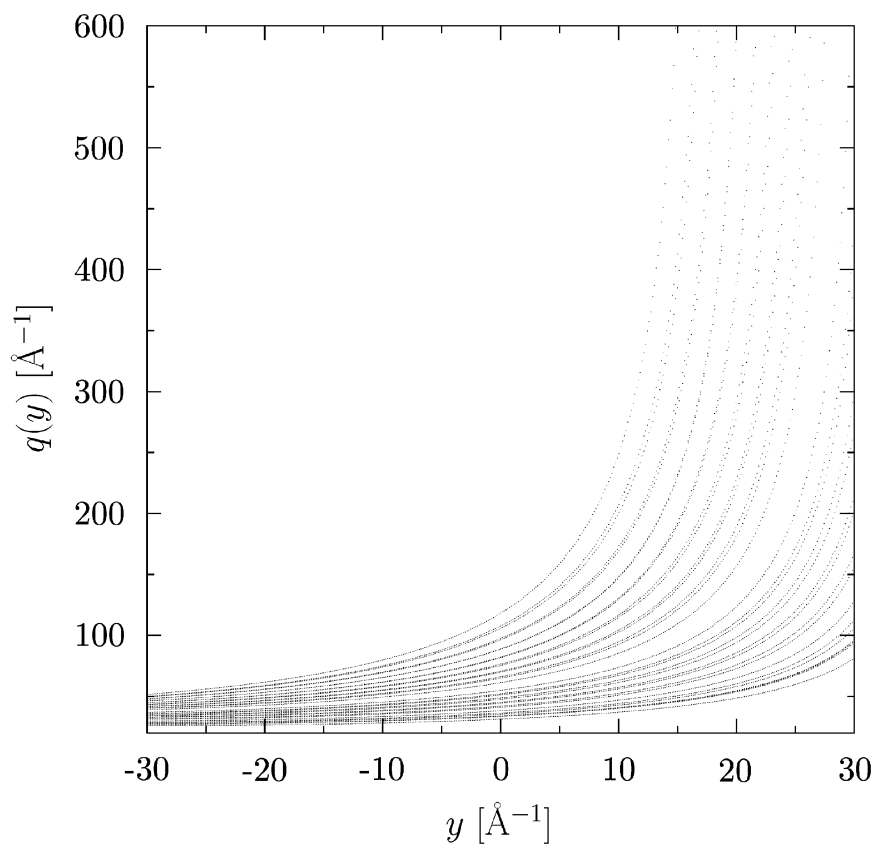

Fig. 4. Fixed-angle $q(y)$ values for proton DINS experiments for a set of typical detector setup covering the range $32.0^{\circ} \leqslant \phi \leqslant 67.5^{\circ}$.

$J(y, q)=J(y)+\Delta J(y, q)$. DINS studies at high wave vector transfers, e.g., $q \geqslant 30 \AA^{-1}$, on proton containing systems $\Delta J(y, q)$ is well accounted for by the expression [17]:

$\Delta J(y, q)=A_{3} \frac{\exp \left(-\left(y^{2} / 2 \sigma^{2}\right)\right)}{\sqrt{2 \pi \sigma^{2}}} \frac{1}{q} H_{3}\left(\frac{y}{\sqrt{2} \sigma}\right)$

where $\mathrm{H}_{3}$ is the third Hermite polynomial and $\sigma$ is the standard deviation of $J(y)$, and the coefficient $A_{3}$ is typically of the order of $0.02 \AA^{-1}$. Thus the sum rules, including the
FSE additive term are

$\int_{-\infty}^{\infty} \mathrm{d} y J(y, q)=1$

$\int_{-\infty}^{0} \mathrm{~d} y J(y, q)=\frac{1}{2}+A_{3} \frac{2}{\sqrt{\pi} q}$

which follow from

$\int_{-\infty}^{\infty} \mathrm{d} y \frac{\exp \left(-\left(y^{2} / 2 \sigma^{2}\right)\right)}{\sqrt{2 \pi \sigma^{2}}} A_{3} \frac{1}{q} H_{3}\left(\frac{y}{\sqrt{2} \sigma}\right)=0$
$\int_{-\infty}^{0} \mathrm{~d} y \frac{\exp \left(-\left(y^{2} / 2 \sigma^{2}\right)\right)}{\sqrt{2 \pi \sigma^{2}}} A_{3} \frac{1}{q} H_{3}\left(\frac{y}{\sqrt{2} \sigma}\right)=A_{3} \frac{2}{\sqrt{\pi} q}$.

We stress that the normalizations above are independent of the particular line shape parameters of $J(y, q)$. These normalizations remain valid in the presence of a broadening due to the convolution of a generic normalized spectrometer resolution function, $R(y, q)$, taking into account the distributive property of the convolution:

$$
\begin{aligned}
& \int_{-\infty}^{\infty} \mathrm{d} y J(y, q) \otimes R(y, q) \\
& \quad=\int_{-\infty}^{\infty} \mathrm{d} y J(y) \otimes R(y, q)+\int_{-\infty}^{\infty} \mathrm{d} y \Delta J(y, q) \otimes R(y, q)=1 \\
& \int_{-\infty}^{0} \mathrm{~d} y J(y, q) \otimes R(y, q)=\int_{-\infty}^{0} \mathrm{~d} y J(y) \otimes R(y, q) \\
& \quad+\int_{-\infty}^{0} \mathrm{~d} y \Delta J(y, q) \otimes R(y, q)=\frac{1}{2}+A_{3} \frac{2}{\sqrt{\pi} q} .
\end{aligned}
$$

In the case of fixed-angle spectra, $q$ varies across the recoil peak, as shown in Fig. 4. Therefore, a proper normalization has to be accounted for, considering that the main effect is to have a $1 / q$ term that is not constant across each of the fixed-angle spectra. For a typical detector arrangement on VESUVIO for proton studies, the fixed-angle $1 / q(y)$ values are reported in Fig. 5. A normalization of the experimental $F^{(\phi)}(y, q(y))$ can be carried out considering that the latter is the sum of an asymptotic part, $F^{(\phi)}(y)$, associated to $J(y)$ and a $q$-dependent part, $\Delta F^{(\phi)}(y, q(y))$, associated to $\Delta J(y, q(y))$. In order to consider the effect of $y$-dependent $q$ values, we report in Fig. 6, the values of two fixed-angle FSE terms, $\Delta^{(\phi)} J(y, q(y))$ together with the

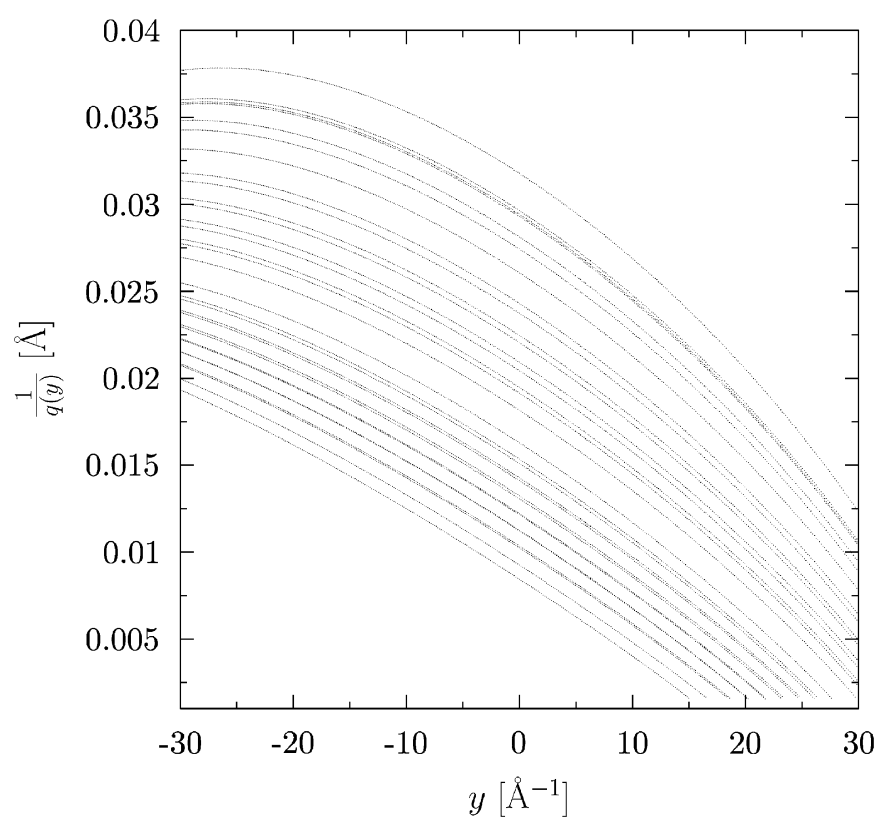

Fig. 5. Fixed-angle $1 / q(y)$ values for proton DINS experiments for a set of typical detector setup covering the range $32.0^{\circ} \leqslant \phi \leqslant 67.5^{\circ}$. 


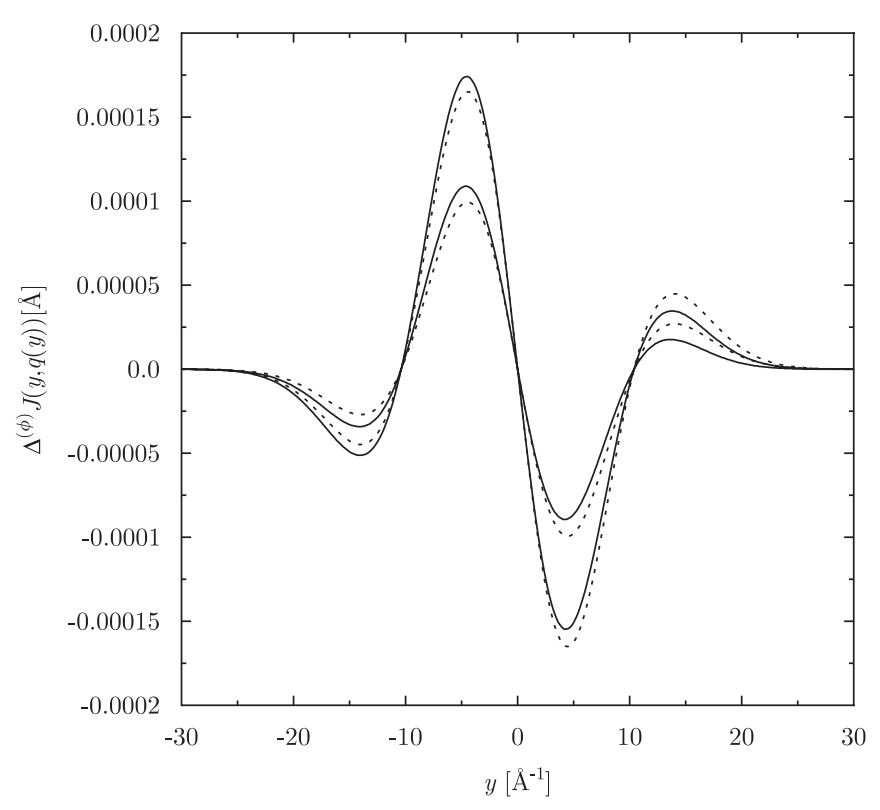

Fig. 6. Fixed-angle $\Delta^{(\phi)} J(y, q(y))$ values for proton DINS experiments for a sample of bulk water at $300 \mathrm{~K}$ (continuous lines). The two angles are $34.1^{\circ}$ and $48.1^{\circ}$, respectively. The corresponding "constant- $q$ " terms, evaluated with $q$ at the center of the recoil peak with values $q^{34^{\circ}}(0)=31.5 \AA^{-1}$ and $q^{48^{\circ}}(0)=52.2 \AA^{-1}$ are reported as dashed lines.

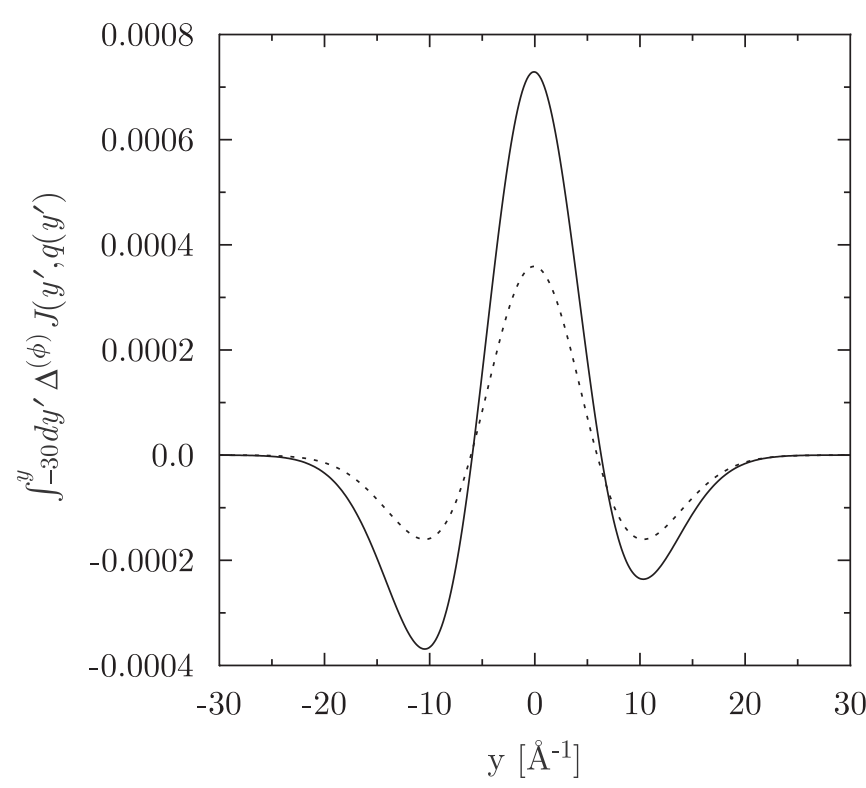

Fig. 7. Numerical integration of $\Delta^{(\phi)} J(y, q(y))$ values for proton DINS experiments for a sample of bulk water at $300 \mathrm{~K}$ (continuous line) for the detector at $34.1^{\circ}$. The corresponding constant- $q$ term, evaluated with $q$ at the center of the recoil peak with a value $q^{34^{\circ}}(0)=31.5 \AA^{-1}$ is reported as a dashed line.

corresponding constant- $q$ terms, evaluated with $q$ at the center of the recoil peak, i.e., $q(0)$ for a sample of bulk water at $300 \mathrm{~K}$. From this figure it can be noted that while the constant- $q$ FSE term, $\Delta^{(\phi)} J(y, q(0))$, is antisymmetric, the corresponding $\Delta^{(\phi)} J(y, q(y))$ is not. The effects of modulation of the $1 / q(y)$ terms are relevant for the normalization of $\Delta^{(\phi)} J(y, q(y))$. A comparison of numerical integration of fixed-angle $\Delta^{(\phi)} J(y, q(y))$ and the equivalent constant- $q \Delta^{(\phi)} J(y, q(0))$ are reported in Figs. 7 and 8. From these two figures we note that the normalization of fixed-angle FSE term holds, the "constant $q$ " and fixed-angle integrals being the same within an uncertainty of $1.0 \times 10^{-5}$ for the integration range

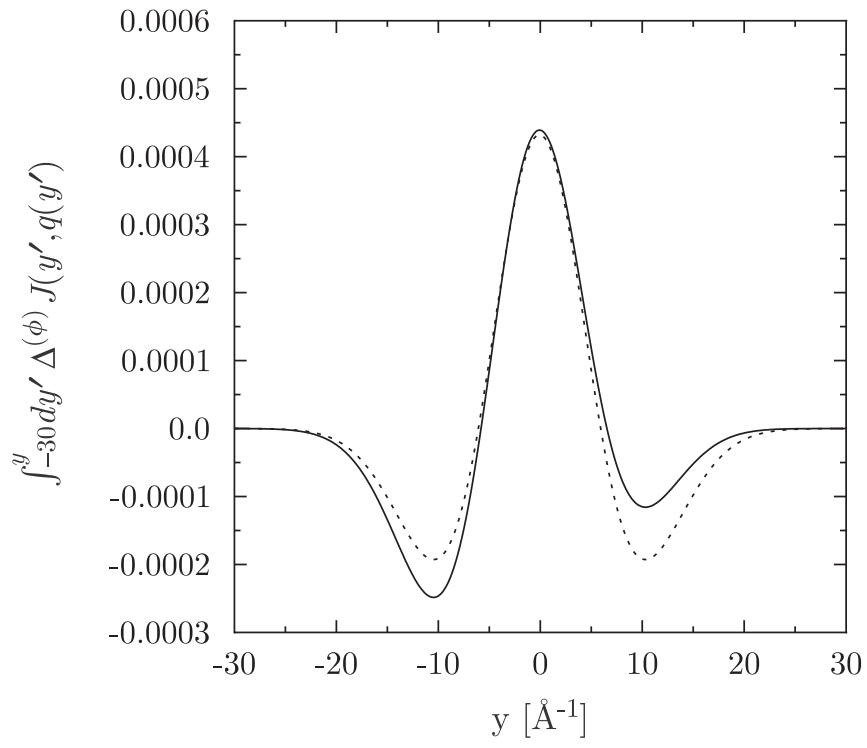

Fig. 8. Numerical integration of $\Delta^{(\phi)} J(y, q(y))$ values for proton DINS experiments for a sample of bulk water at $300 \mathrm{~K}$ (continuous line) for the detector at $48.1^{\circ}$. The corresponding constant $q$-term, evaluated with $q$ at the center of the recoil peak with a value $q^{48^{\circ}}(0)=52.2 \AA^{-1}$ is reported as a dashed line.

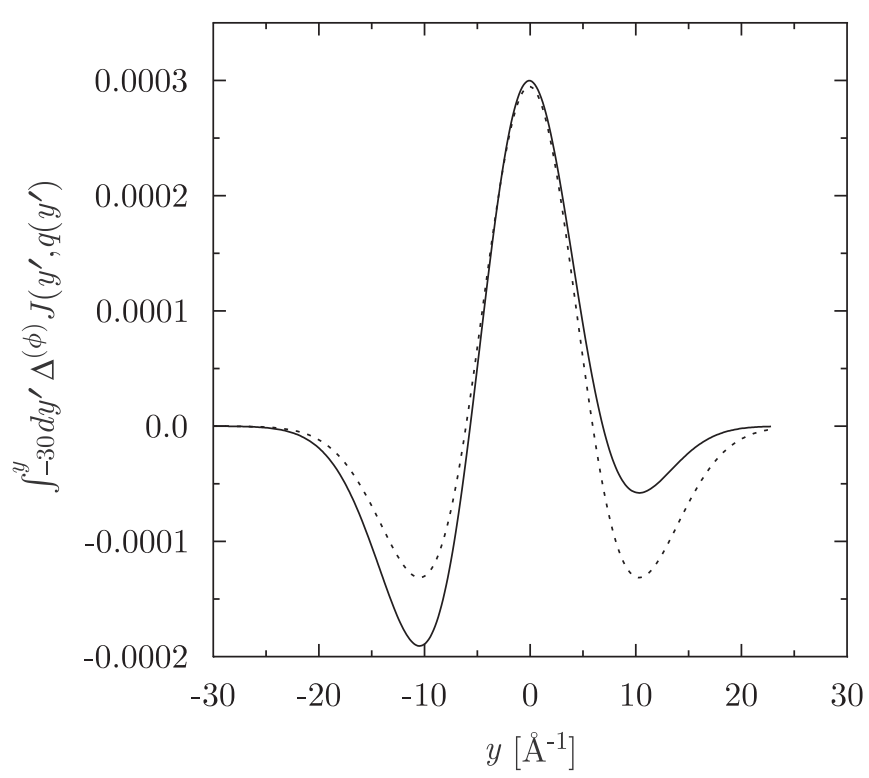

Fig. 9. Numerical integration of $\Delta^{(\phi)} J(y, q(y))$ values for proton DINS experiments for a sample of bulk water at $300 \mathrm{~K}$ (continuous line) for the detector at $57.5^{\circ}$. The corresponding constant- $q$ term, evaluated with $q$ at the center of the recoil peak with a value $q^{57^{\circ}}(0)=76.5 \AA^{-1}$ is reported as a dashed line.

between -30 and $30 \AA^{-1}$. In the case of scattering angles above approximately $50^{\circ}$ the upper limit of $y, y_{\max }$ is lower than $30 \AA^{-1}$ with upper values of $q \simeq 600 \AA^{-1}$ [24]. For example, for $\phi=57.5^{\circ}$, $y_{\max }=23.1 \AA^{-1}$, while for $\phi=67.5^{\circ}, y_{\max }=15.1 \AA^{-1}$. At these high scattering angles, the lower limit of $y$ remains $-30 \AA^{-1}$. The normalization to be used in this case is

$\int_{-\infty}^{0} \mathrm{~d} y J(y, q)=\frac{1}{2}+A_{3} \frac{2}{\sqrt{\pi} q}$.

Numerical integration of fixed-angle $\Delta^{(\phi)} J(y, q(y))$ and the equivalent constant- $q \Delta^{(\phi)} J(y, q(0))$ with the normalization of Eq. (5.6) are reported in Figs. 9 and 10. In the latter, the constant- $q$ and fixedangle integrals have a maximum difference of $2.6 \times 10^{-4}$ for the 


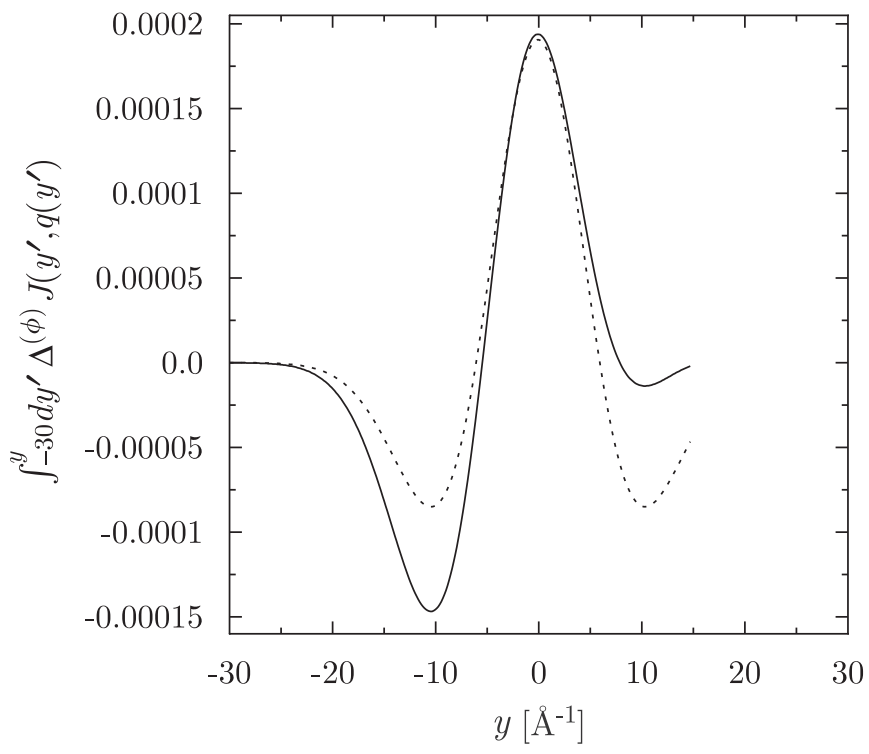

Fig. 10. Numerical integration of $\Delta^{(\phi)} J(y, q(y))$ values for proton DINS experiments for a sample of bulk water at $300 \mathrm{~K}$ (continuous line) for the detector at $67.5^{\circ}$. The corresponding constant- $q$ term, evaluated with $q$ at the center of the recoil peak with a value $q^{67^{\circ}}(0)=118.4 \AA^{-1}$ is reported as a dashed line.

integration range between -30 and $0 \AA^{-1}$. Thus, even the limited $y$-range spectra can be normalized with a maximum error of $0.05 \%$.

The whole set of fixed-angle experimental Neutron Compton Profiles, $F^{(\phi)}(y, q(y))$, can be, therefore, normalized independently of individual detector element calibration, thanks to the zeroorder sum rules outlined in Eq. (5.3). We stress that this procedure can be applied specifically for studies of line shapes of Neutron Compton Profiles. Being independent of the absolute scattering intensities, it is not suitable for studies of proton scattering crosssections.

\section{Constant- $q$ rebinning procedure}

Constant- $q$ spectra on inelastic time-of-flight spectrometers are usually obtained either by interpolation or by rebinning procedures. For rebinning procedures, the normalized $F^{(\phi)}(y, q(y))$ spectra provide a rectangular $[y, q(y)]$ grid that has to be inspected for the choice of suitable $q$ values. The useful range of constant- $q$ values for the extraction of constant- $q$ spectra can be estimated, for example, from Fig. 4 . The $q(y)$ values shown are "denser" in the $q$ range $30 \AA^{-1} \leqslant q \leqslant 70 \AA^{-1}$. The useful choice of fixed- $q$, therefore, lies in the above range, as shown in Fig. 11. The $q$ width onto which the data have to be rebinned is chosen to be larger than the $q$ uncertainty, $\Delta q(y)$, for each fixed-angle detector [26]. A typical $q(y)$ uncertainty, for proton neutron Compton scattering, is of the order of $2.5 \AA^{-1}$ (half width) [27]. In Fig. 12, the values of $\Delta q(y)$ as a function of $q(y)$ for each fixed-angle detector element are reported. It can be noticed that the constant- $q$ widths can be chosen with a value of at least $\Delta q= \pm 2.5 \AA^{-1}$. As an example, we can consider a rectangular $[y, q(y)]$ grid centered at $q=$ $(53 \pm 3) \AA^{-1}$, as shown in Fig. 13. Approximately 800 data points are in the grid and contribute to produce a constant- $q$ spectrum in the range $-25 \AA^{-1} \leqslant y \leqslant 25 \AA^{-1}$. A blow-up in the range $5 \AA^{-1} \leqslant y \leqslant 20 \AA^{-1}$ is reported in Fig. 14. For example, in the $y$ bin centered at $9 \AA^{-1}$, three data points can be rebinned, while in the $y$ bin centered at $18 \AA^{-1}$, four data points can be rebinned. Therefore, for each $y_{i}$ bin the constant- $q$ value of the experimental

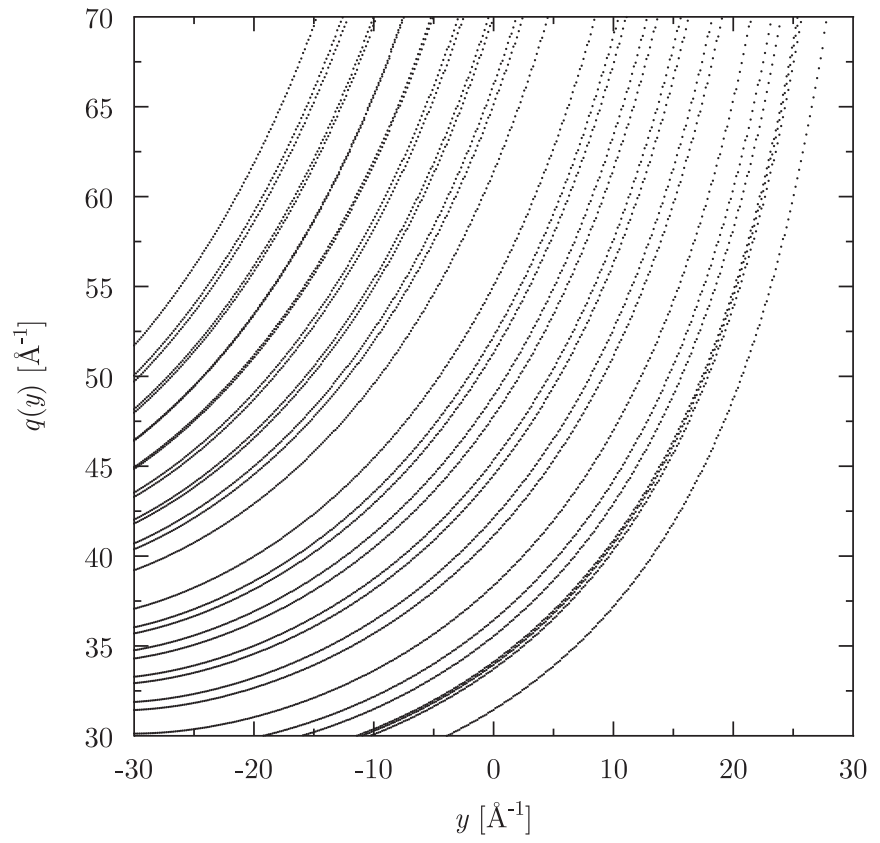

Fig. 11. Fixed-angle $q(y)$ values, in the range $30 \AA^{-1} \leqslant q \leqslant 70 \AA^{-1}$, for proton DINS experiments for a set of typical detector setup covering the range $32.0^{\circ} \leqslant \phi \leqslant 67.5^{\circ}$.

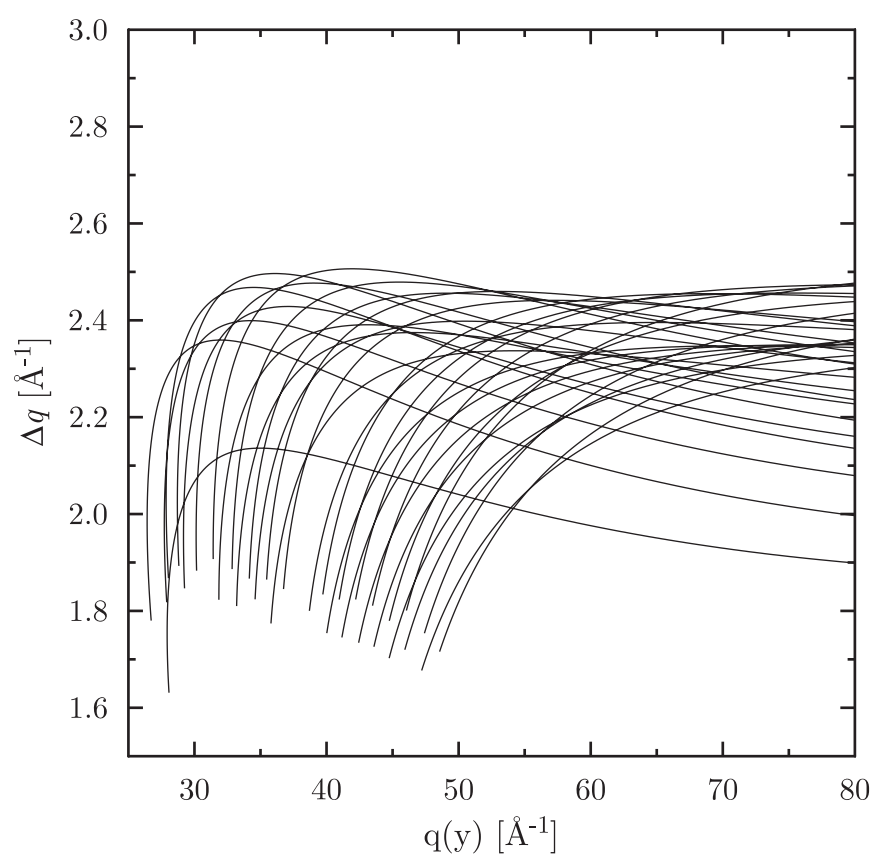

Fig. 12. Values of $\Delta q(y)$ as a function of $q$ for each single-angle detector element for proton DINS experiments for a set of typical detector setup covering the range $32.0^{\circ} \leqslant \phi \leqslant 67.5^{\circ}$.

NCP can be derived as

$F\left(y_{i}, q\right)=\frac{\sum_{j=1}^{n} F^{\left(\phi_{j}\right)}\left(y_{i}, q\left(y_{i}\right)\right)}{n}$

where $F^{\left(\phi_{j}\right)}\left(y_{i}, q\left(y_{i}\right)\right)$ is the $j$-th fixed-angle experimental Neutron Compton Profile value in the $y_{i}$ bin at the chosen $q\left(y_{i}\right)$ value in the grid. The number of points in the chosen $y$ bin, $n$, is typically between three and four, as shown above. Standard error propagation is then carried out to determine the experimental uncertainty on each $F\left(y_{i}, q\right)$ value. The corresponding constant- $q$ 


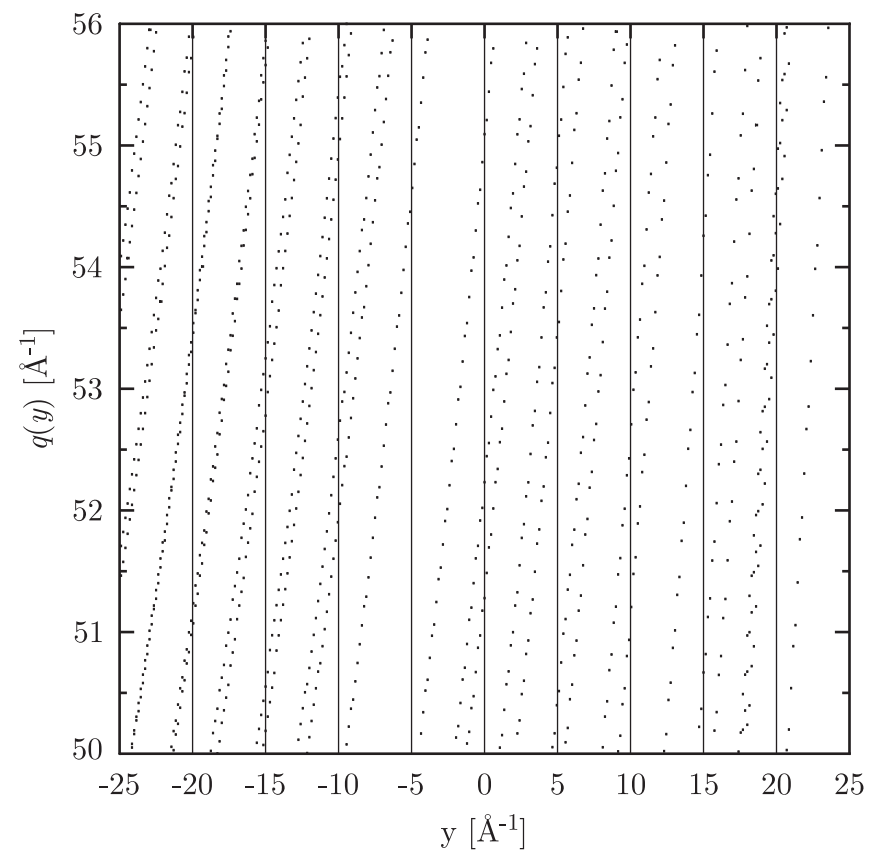

Fig. 13. Rectangular $y, q(y)$ grid centered at $q=(53 \pm 3) \AA^{-1}$ (dots). The vertical continuous lines are a guide for the eyes.

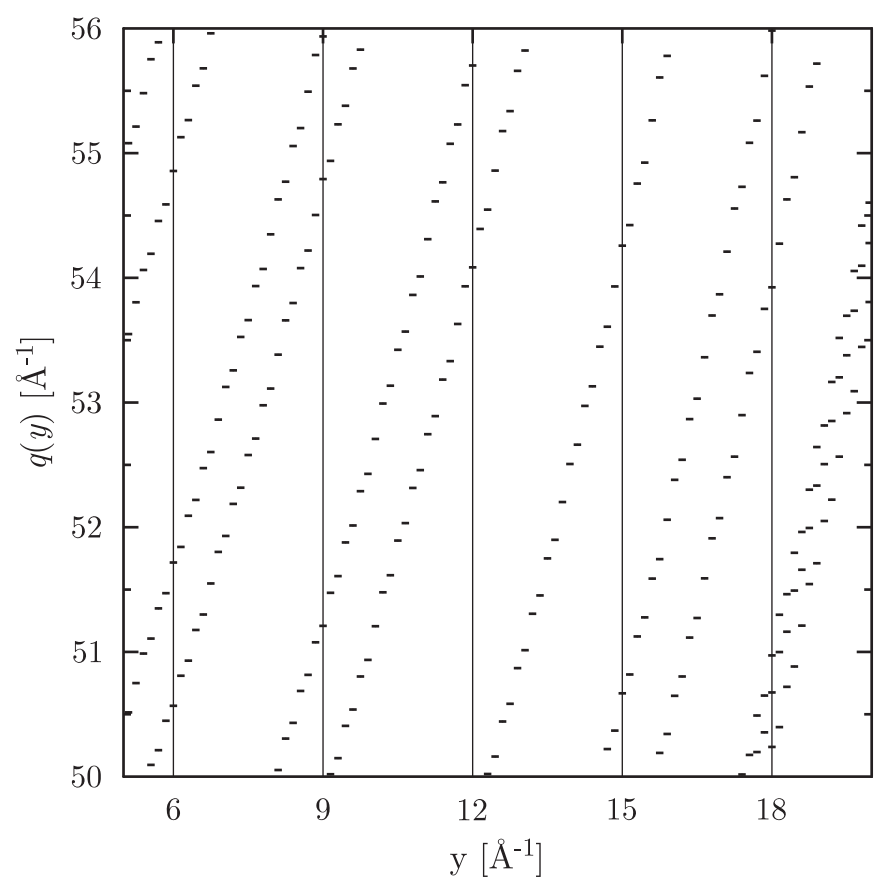

Fig. 14. Rectangular $y, q(y)$ grid centered at $q=(53 \pm 3) \AA^{-1}$ (dots) in the range $5 \AA^{-1} \leqslant y \leqslant 20 \AA^{-1}$. The horizontal bars denote the typical width of a $y$ bin of $0.15 \AA^{-1}$. The vertical continuous lines are a guide for the eyes.

resolution functions are calculated accordingly (the distributive property of convolution being still applicable):

$R\left(y_{i}, q\right)=\frac{\sum_{j=1}^{n} R^{\left(\phi_{j}\right)}\left(y_{i}, q\left(y_{i}\right)\right)}{n}$.

Examples of constant- $q$ experimental Neutron Compton Profiles, $F(y, q)$, together with the corresponding resolutions, $R(y, q)$, evaluated at the following $q$ values, $(53 \pm 3),(60 \pm 4)$ and $(40 \pm 3) \AA^{-1}$, are reported in Figs. 15-17, respectively. The rebin-

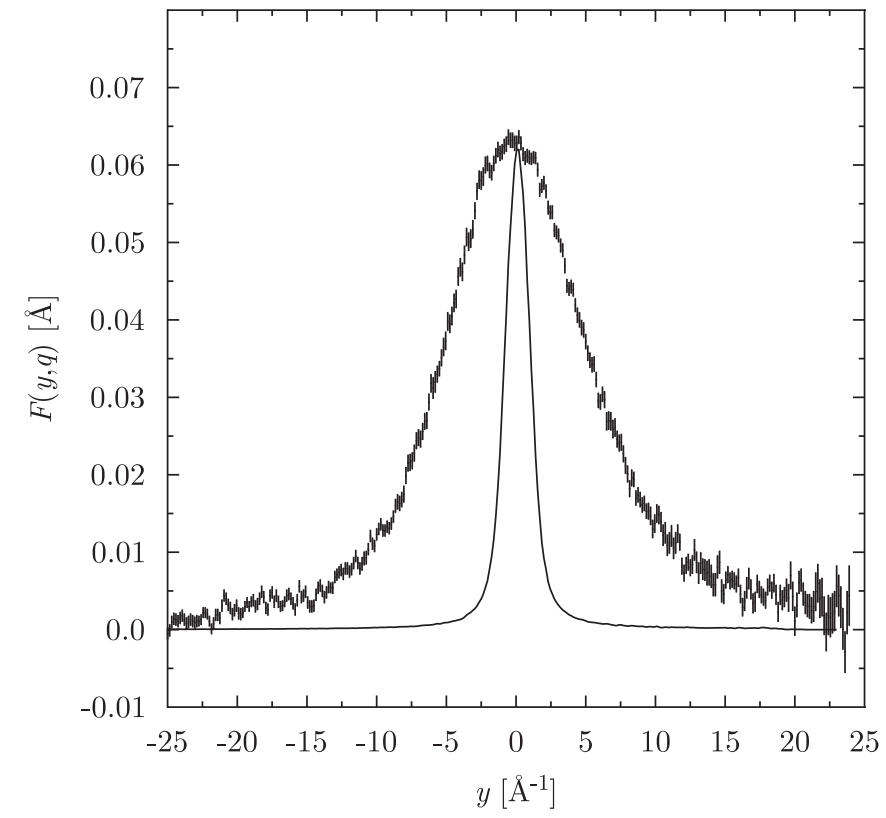

Fig. 15. Constant- $q$ experimental Neutron Compton Profile, $F(y, q)$ (dots with error bars), together with the corresponding resolution, $R(y, q)$, normalized to peak height (continuous line) for $q=(53 \pm 3) \AA^{-1}$.

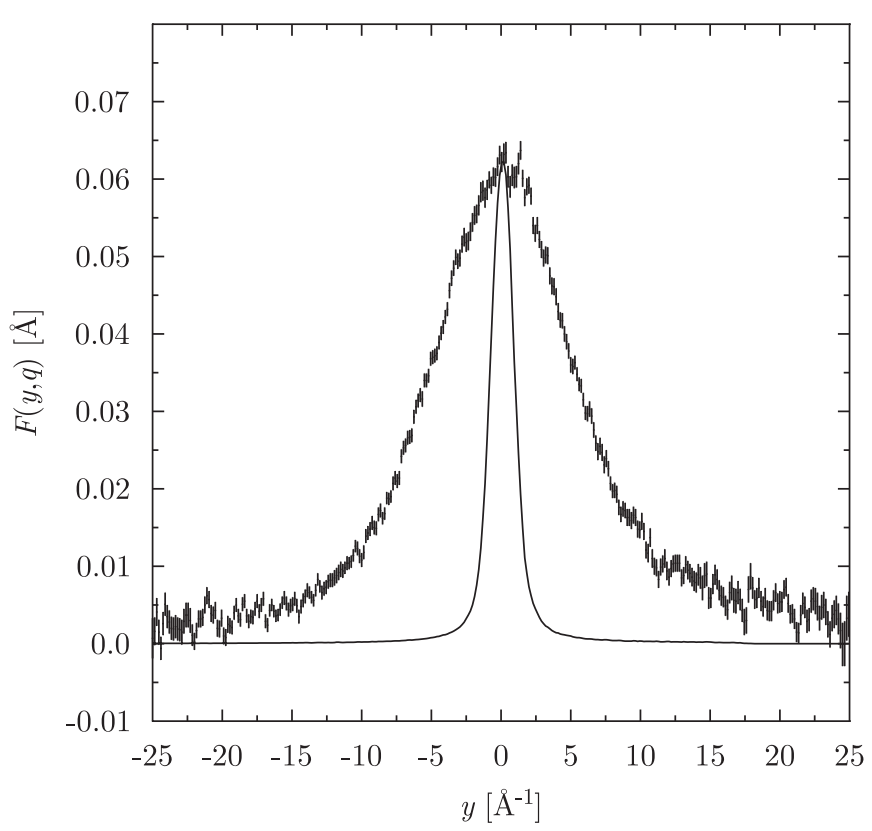

Fig. 16. Constant- $q$ experimental Neutron Compton Profile, $F(y, q)$ (dots with error bars), together with the corresponding resolution, $R(y, q)$, normalized to peak height (continuous line) for $q=(60 \pm 4) \AA^{-1}$.

ning algorithm has also the option of searching the most frequent $q$-value in the grid, reconstructing the corresponding spectrum. In the present example of room temperature water, the most frequent $q$-value is $(35 \pm 3) \AA^{-1}$, and the corresponding constant- $q$ spectrum is reported in Fig. 18.

\section{Discussion and conclusions}

From Figs. 15-18, it is possible to note that the magnitude of the error bars (and noise) of the constant- $q$ Neutron Compton 


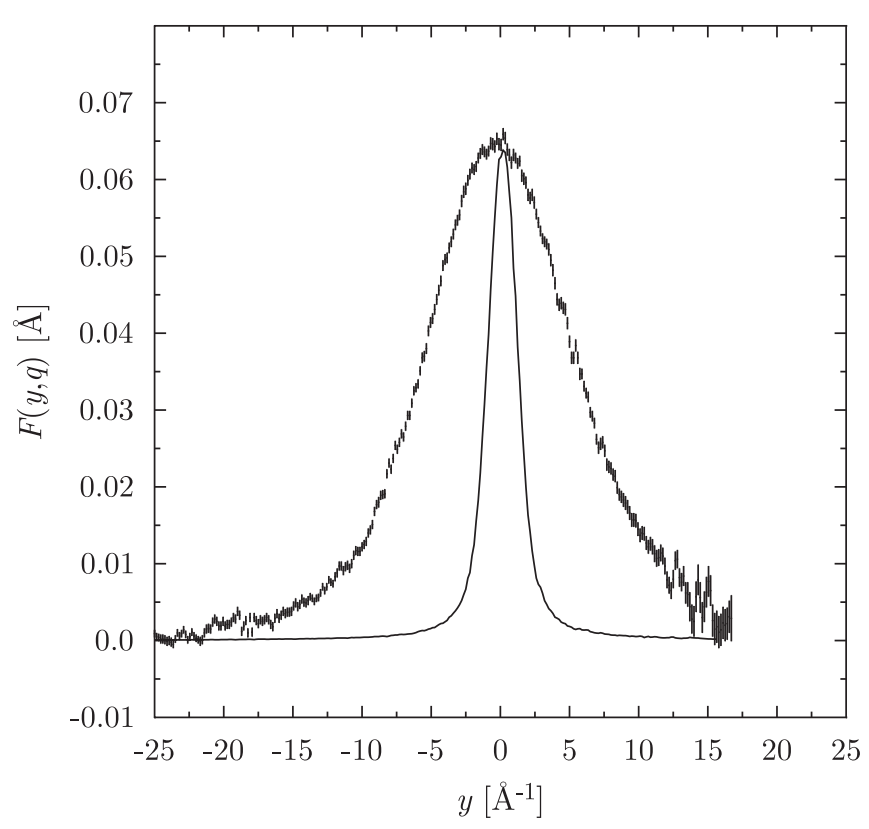

Fig. 17. Constant- $q$ experimental Neutron Compton Profile, $F(y, q)$ (dots with error bars), together with the corresponding resolution, $R(y, q)$, normalized to peak height (continuous line) for $q=(40 \pm 3) \AA^{-1}$.

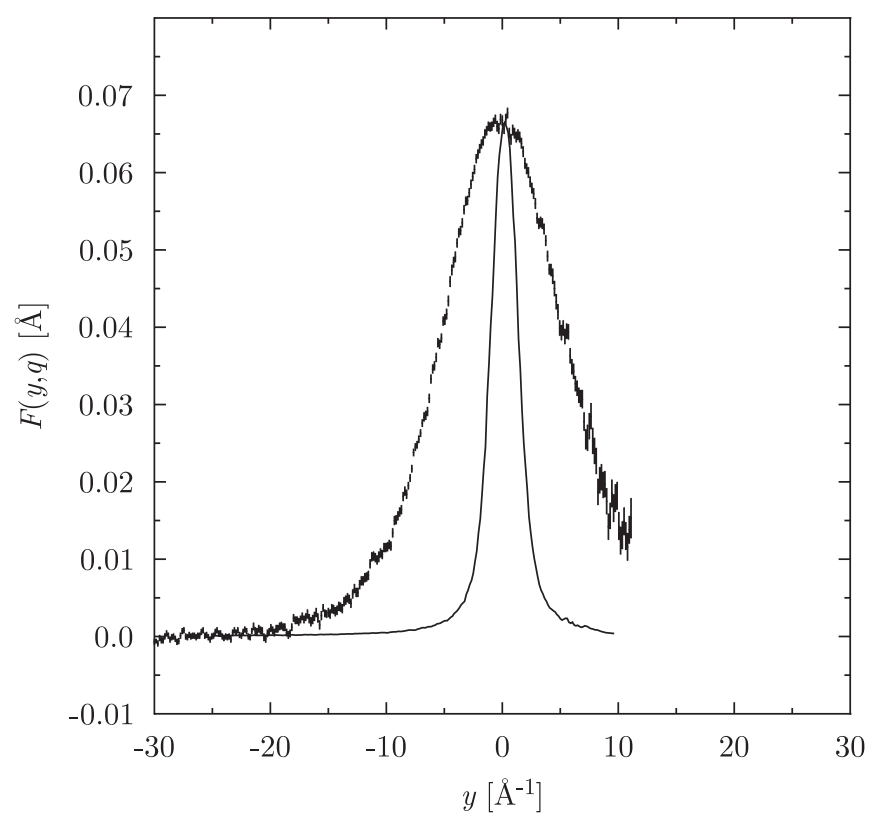

Fig. 18. Constant- $q$ experimental Neutron Compton Profile, $F(y, q)$ (dots with error bars), together with the corresponding resolution, $R(y, q)$, normalized to peak height (continuous line) for the most frequent $q$-value, $q=(35 \pm 3) \AA^{-1}$.

Profiles is considerably reduced, as compared to fixed-angle spectra. This has great advantages for the determination of line shape parameters, especially for the non-Gaussian components in the longitudinal momentum distribution. Although in some cases the resulting spectrum has a limited $y$ range, for example, in the positive $y$ tails, the negative $y$ tails have always much smaller errors and noise as compared to fixed-angle spectra. In the case of high- $q$ cuts, such as $q=60 \AA^{-1}$, the positive $y$ tails show a very small noise as compared, for example, to the fixed-angle spectrum reported in Fig. 3. Clearly, the high wave vector transfer data points, e.g., $q \geqslant 70 \AA^{-1}$, are excluded from the spectra. The latter are generally affected by larger noise since, for the present experimental setup and given the primary and secondary flight path lengths, they appear in time-of-flight regions below $100 \mu \mathrm{s}$, where data collection is more affected by noise. We envisage that determination of line shape parameters of momentum distribution can be carried out by comparing the analysis of fixed-angle as well as constant- $q$ data sets. This approach has been recently applied for proton momentum distribution in hydrated lysozyme [10] and in water confined in nanoporous xerogel [9]. The two approaches yielded the same momentum distribution line shape, but the constant- $q$ data analysis provided smaller uncertainties on the line shape parameters. Moreover, a constant- $q$ representation of DINS data is more amenable for comparison with theoretical predictions, being independent of the particular kinematic $(q, \omega)$ path of the fixed-angle detectors data.

In conclusion, a procedure to collect scattering signals from different fixed-angle detectors to obtain Neutron Compton Profiles at constant wave vector transfer, $q$, has been presented and applied for the case of proton Neutron Compton Profile measurements on the VESUVIO spectrometer at the ISIS pulsed neutron source. This relies on the normalization of fixed-angle Neutron Compton Profiles, that allows signals from different detectors to be compared and rebinned by specifically developed procedure. The latter involves two main approximations, whose validity has been carefully tested and assessed in numerous measurements on VESUVIO: (1) the Convolution Approximation; (2) the $1 / q$ series expansion of the Final State Effects. This procedure can be applied specifically for studies of line shapes of Neutron Compton Profiles, being independent of the absolute scattering intensities. For possible future Deep Inelastic Neutron Scattering spectrometers, a wider solid angle coverage with improved angular resolution, which would be possible by making use of the resonant detector configuration with YAP scintillators [14,15], would greatly enhance capability of the method described here.

\section{Acknowledgments}

The authors acknowledge stimulating scientific discussions with J. Mayers (ISIS, Rutherford Appleton Laboratory, UK). This work was supported within the CNR-CCLRC Agreement no. $01 / 9001$ concerning collaboration in scientific research at the spallation neutron source ISIS. The financial support of the Consiglio Nazionale delle Ricerche in this research is hereby acknowledged.

\section{References}

[1] C. Andreani, D. Colognesi, J. Mayers, G.F. Reiter, R. Senesi, Adv. Phys. 54 (2005) 377.

[2] G.I. Watson, J. Phys. Condens. Matter 8 (1996) 5955.

[3] R. Senesi, C. Andreani, Z. Bowden, D. Colognesi, E. Degiorgi, A.L. Fielding, J. Mayers, M. Nardone, J. Norris, M. Praitano, N.J. Rhodes, W.G. Stirling, J. Tomkinson, C. Uden, Phys. B Condens. Matter 276 (2000) 200.

[4] J. Mayers, J. Tomkinson, T. Abdul-Redah, W.G. Stirling, C. Andreani, R. Senesi, M. Nardone, D. Colognesi, E. Degiorgi, Physica B Condens. Matter 350 (2004) E659.

[5] M.A. Adams, J. Mayers, O. Kirichek, R.B.E. Down, Phys. Rev. Lett. 98 (2007) 085301.

[6] C. Andreani, C. Pantalei, R. Senesi, Phys. Rev. B 75 (2007) 064515.

[7] C. Andreani, C. Pantalei, R. Senesi, J. Phys. Condens. Matter 18 (2006) 5587.

[8] G. Reiter, C. Burnham, D. Homouz, P.M. Platzman, J. Mayers, T. Abdul-Redah, A.P. Moravsky, J.C. Li, C.-K. Loong, A.I. Kolesnikov, Phys. Rev. Lett. 97 (2006) 247801.

[9] V. Garbuio, C. Andreani, S. Imberti, A. Pietropaolo, G.F. Reiter, R. Senesi, M.A. Ricci, J. Chem. Phys. 127 (2007) 4501.

[10] R. Senesi, A. Pietropaolo, A. Bocedi, S.E. Pagnotta, F. Bruni, Phys. Rev. Lett. 98 (2007) 138102.

[11] D. Homouz, G. Reiter, J. Eckert, J. Mayers, R. Blinc, Phys. Rev. Lett. 98 (2007) 115502.

[12] D. Colognesi, A. Pietropaolo, R. Senesi, A.J. Ramirez-Cuesta, Phys. Rev. B 76 (2007) 174206 
[13] A. Pietropaolo, D. Fernandez-Cañoto, E. Perelli-Cippo, S. Dirè, P. Prosposito, Phys. Rev. B 77 (2008) 014202.

[14] E.M. Schooneveld, J. Mayers, N.J. Rhodes, A. Pietropaolo, C. Andreani, R. Senesi, G. Gorini, E. Perelli-Cippo, M. Tardocchi, Rev. Sci. Inst. 77 (2006) 5103.

[15] C. Andreani, A. Pietropaolo, R. Senesi, G. Gorini, E. Perelli-Cippo, M. Tardocchi, N. Rhodes, E.M. Schooneveld, Appl. Phys. Lett. 85 (2004) 5454

[16] A. Pietropaolo, C. Andreani, A. Filabozzi, E. Pace, R. Senesi, Nucl. Instr. and Meth. Phys. Res. A 570 (2007) 498.

[17] V.F. Sears, Phys. Rev. B 30 (1984) 44.

[18] S.O. Diallo, J.V. Pearce, R.T. Azuah, H.R. Glyde, Phys. Rev. Lett. 93 (2004) 075301.

[19] S.O. Diallo, J.V. Pearce, R.T. Azuah, O. Kirichek, J.W. Taylor, H.R. Glyde, Phys. Rev. Lett. 98 (2007) 205301.
[20] R. Senesi, D. Colognesi, A. Pietropaolo, T. Abdul-Redah, Phys. Rev. B 72 (2005) 054119.

[21] J. Mayers, A.L. Fielding, R. Senesi, Nucl. Instr. and Meth. Phys. Res. A 481 (2002) 454.

[22] R. Senesi, C. Andreani, D. Colognesi, J. Low Temp. Phys. 126 (2002) 57.

[23] M. Krzystyniak, C.A. Chatzidimitriou-Dreismann, Phys. Rev. B 72 (2005) 174117.

[24] R.A. Cowley, J. Mayers, J. Phys. Condens. Matter 18 (2006) 5291.

[25] B. Dorner, Nucl. Instr. and Meth. Phys. Res. B 247 (2006) 390.

[26] K.H. Andersen, W.G. Stirling, R. Scherm, A. Stunault, B. Fak, H. Godfrin, A.J. Dianoux, J. Phys. Condens. Matter 6 (1994) 821.

[27] S. Imberti, C. Andreani, V. Garbuio, G. Gorini, A. Pietropaolo, R. Senesi M. Tardocchi, Nucl. Instr. and Meth. Phys. Res. A 522 (2005) 463. 